\title{
Testing of Goose Protocol of IEC61850 Standard in Protection IED
}

\author{
Chilton Fernandes \\ Microelectronics, ETC \\ dept.,Goa College of \\ Engineering, Goa university, \\ India.
}

\author{
Samarth Borkar \\ Professor, ETC dept, \\ Goa College of Engineering, \\ Goa University India
}

\author{
Jignesh Gohil \\ Developer-firmware \\ Siemens Itd
}

\begin{abstract}
High speed communication between IED to IED is required for successful implementation of protection schemes in a substation automation system. IEC 61850 is an International standard that supports high speed peer-to-peer services like GOOSE. The communication using GOOSE protocol replaces point to point copper wiring between the IEDs in conventional substations with high speed communication over Ethernet technology. Individual IED needs to be tested before they are installed in the field. In this paper the practical setup for testing of protection schemes of an IED and its conformity to GOOSE protocol of IEC 61850 standard is presented. Brief introduction to the GOOSE protocol and its advantages is presented in the paper.
\end{abstract}

\section{Keywords}

IEC 61850, GOOSE, protection, SAS

\section{INTRODUCTION}

The IEC 61850 standard for substation enables the integration of all protection, control, measurement and monitoring functions. These functions require high speed and reliable communication in the substation. Traditionally this was achieved using copper wires between the control center and the IEDs and also between the IEDs in the substation. Using copper wire for communication creates a very complex network of wires inside the substation. Now Ethernet based local area network (LAN) provides better speed, reliability and is easy to install and maintain. Generic Object Oriented Substation Events (GOOSE) works on Ethernet for communication between intelligent electronic devices (IED) to IED [3].

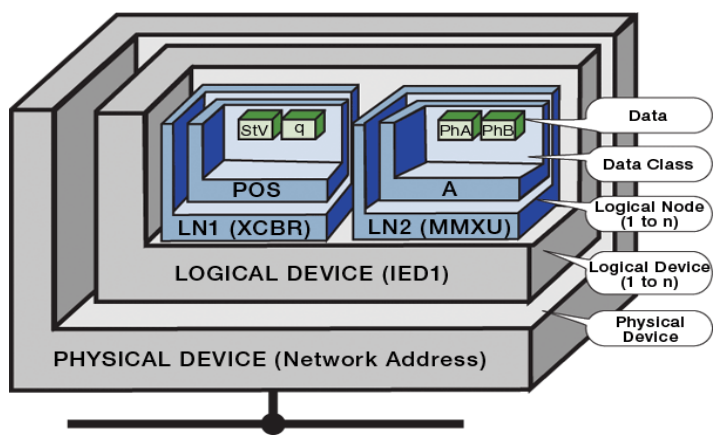

Figure 1: Data model of IEC61850

IEC61850 standard was introduced to achieve interoperability amongst the substation devices from different vendor. To achieve this, a modular, hierarchical data model was defined as shown in figure $1[1]$.
Any object in the IEC61850 standard is referenced as follows:

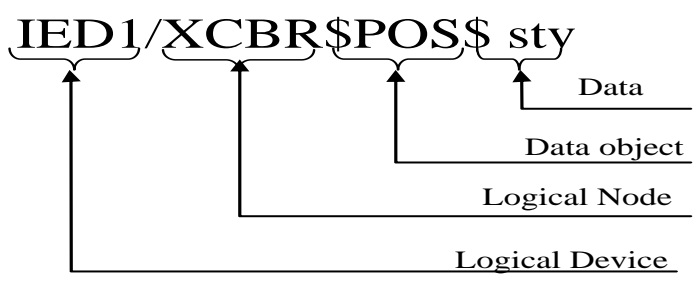

Figure 2: Object naming in IEC61850

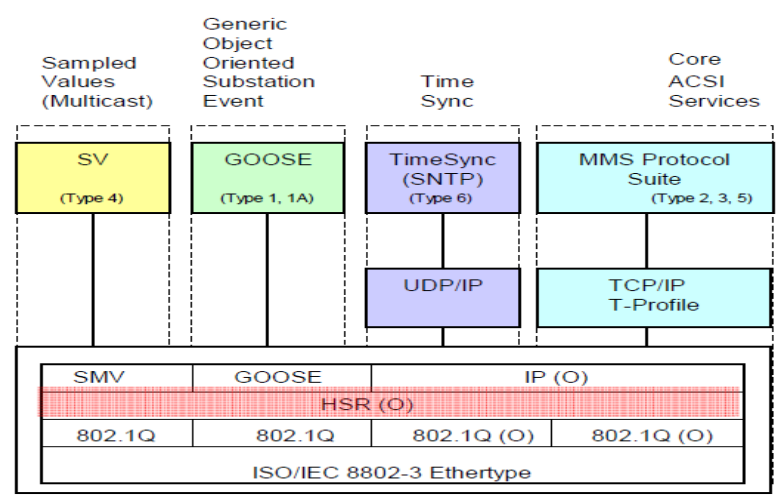

Figure 3: Communication profiles of protocols used in IEC61850

The communication profiles used in IEC61850 standard is shown in figure 3 . Here the mapping of the IEC61850 services to the open system interconnection (OSI) layers is shown. The GOOSE service uses three layers of OSI model namely application, data link layer and the physical layer [3].

In this paper we will demonstrate testing of GOOSE functionality in a protection IED. Testing of two such IEDs in the laboratory environment is presented. Brief introduction to the GOOSE protocol data unit (PDU) and analysis of the GOOSE frame is given in the next section.

The setting up of IEDs, its configuration, testing and the tools required are listed in the experimental setup section. The test performed in the paper is to ensure that the IED is capable of publishing GOOSE messages, when a change in event takes place in the substation, and also subscribe to them. The change in event will be simulated using the OMICRON test tools. The other test performed will be to test the round trip communication. 


\section{GOOSE OVERVIEW}

IEC61850-7-2 defines a generic substation event (GSE) service which can provide a fast and reliable distribution of input and output data values, including either digital or analog values [1]. In the IEC 61850-8-1, one of the messages associated with the GSE services is the GOOSE message that allows for multicast messages across the LAN [2].

The GOOSE message frame consists of the following:

\section{The header MAC}

The destination address is defined in the standard of IEC61850. It is a multicast address defined as 01-0C-CD-xx$\mathrm{xx}-\mathrm{xx}$.

The source address is the MAC of the sending device.

\section{Priority tagged}

TPID (Tag Protocol Identifier) Field: Indicates the Ethernet Type assigned for 802.1Q Ethernet encoded frames.

TCI (Tag Control Information) Fields:

User Priority: BS3; User priority value shall be set by configuration to separate sampled values from low priority busload.

VID: Virtual LAN support is optional. If this mechanism will be used, the VLAN Identifier (VID) shall be set by configuration, if it is not used, it shall be set to zero (0).

\section{Ethernet-PDU}

Ethernet Type: In this standard, the GOOSE type is defined 0x88B8.

APPID: application identifier. The APPID is used to select ISO/IEC 8802-3 frames containing GOOSE messages and to distinguish the application association. The value of APPID is the combination of the APPID type, defined as the two most significant bits of the value and the actual ID.

Length: Number of octets including the Ether type PDU header starting at APPID, and the length of the APDU (Application Protocol Data Unit). Therefore, the value of Length shall be $8+m$, where $m$ is the length of the APDU and $m$ is less than 1492 .

The GOOSE APDU consists of the following:

State number (stNum): This number is assigned whenever a GOOSE message is generated as a result of event change.

Sequence number (sqNum): This number is assigned in increasing order to retransmitted GOOSE messages.

Test: Is set if in test mode.

Time allowed to live (TAL): The maximum time packet remains alive after transmission.

NdsCom: Set when the data in the GOOSE message is invalid

Configuration revision (confRev): Indicates the version of the IED. ConfRev of the publisher and the subscriber should be the same.

Number of dataset entries (numDatSetEntries): Indicates the number of data present in the received GOOSE message.

GOOSE control block reference (gocbRef): Gives the name of the GOOSE control block
Data set (datset): Gives the name of the GOOSE dataset in the IED.

GOOSE ID (goID): GOOSE block in each IED has a different ID associated to it.

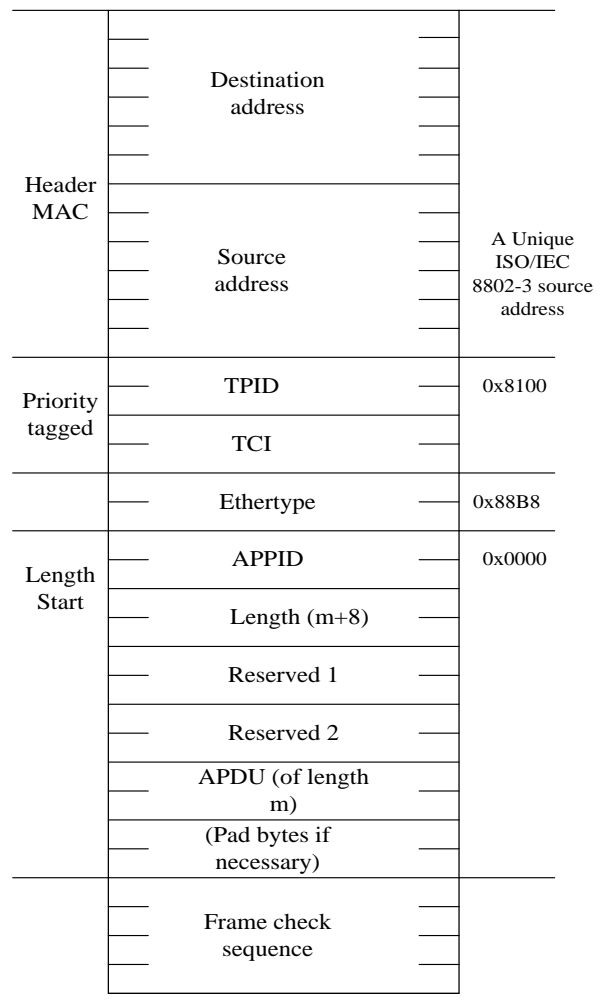

Figure 4: GOOSE frame as per IEC61850-8-1

Timestamp ( $\mathrm{t}$ ): Each GOOSE message is associated the time when it was generated.

Data: Contains the information of the GOOSE message.

\section{ADVANTAGES OF GOOSE PROTOCOL}

- GOOSE protocol uses the standard Ethernet for communication. As Ethernet is evolving, the progress in its development will be reflected on the GOOSE communication and enhance its advantages.

- Ethernet replaces point to point hardwired copper connection between different IEDs.

- GOOSE does not use a handshake mechanism hence speed performance requirements of substation automation functions is improved.

- To ensure highest level of reliability, GOOSE messages are repeated as long as the state persists. These heartbeat messages are sent continuously in the network with a long cycle time which ensures that the devices which have been activated recently, will know the current status values of their peer devices in the network. Each packet has a fixed time allowed to live (TAL) in the network.

- The GOOSE is directly mapped to the Ethernet layer hence the processing time is also very less which is best suited for very time critical protection function in the substation. 
- To maximize dependability and security, GOOSE message has a "hold time" parameter, which defines the time for which the message will live and afterwards will expire unless the same status message is repeated or a new message is received prior to the expiration of the hold time.

- One single GOOSE message, of an individual IED, can contain all the required data related to the protection scheme, whereas the hard-wired approach requires function specific connection, and hence reduces the network traffic during fault conditions.

- GOOSE message is not a command in the sense that it does not tell any receiving device what to do, rather it just indicates that a new event has occurred, what that event is and the time when it happened.

- GOOSE messages also use advanced configuration of Ethernet frames like Virtual local area network (VLAN) and priority tagging. This priority helps to filter out the message especially when there is high traffic, which can occur when a fault occurs.

GOOSE protocol uses multicast mode of communication which enables multiple IEDs to receive the same data at the same time.

\section{EXPERIMENTAL TEST SETUP}

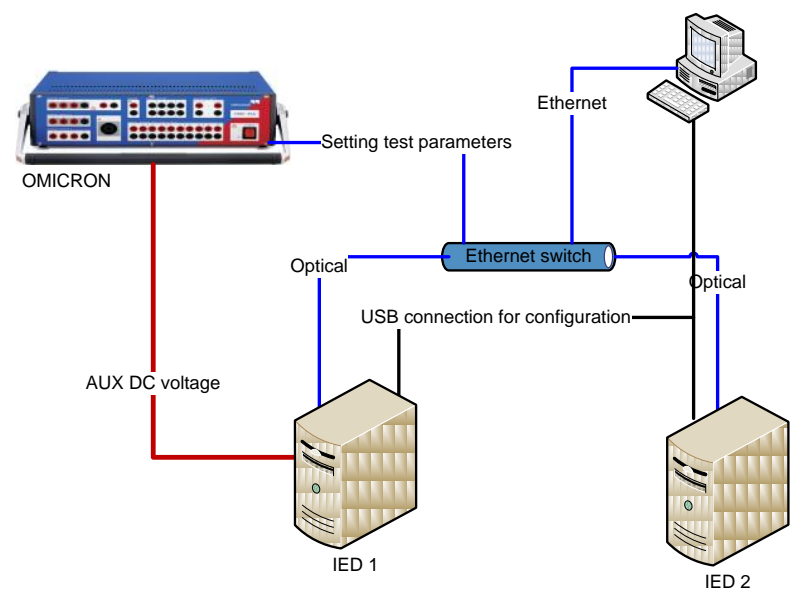

Figure 5: Test setup for GOOSE testing.

Two IEC61850 compliant IEDs are used for testing. Initially the IED is connected to a PC using serial USB connection for initial configuration. Once the IED's Ethernet interface is configured it is connected to an Ethernet switch for communication testing. The Omicron test tool is connected to the PC with an Ethernet connection for setting the test parameters.

The IED is configured using IED configuration manger and the IEC61850 related parameters are set by IEC61850 configurator. Omicron test tool is used to simulate substation event in the laboratory environment.

IED1 and IED2 are connected to an Ethernet switch shown in figure 5. Also there is an USB connection from the IEDs to the PC. Omicron is connected to the PC via the Ethernet switch and to the IED1 via electric cable. This electric cable connection is use to supply current to the current input.

\subsection{Protection IED.}

Protection IED is a device capable of measuring the incoming current input and based on the user settings, calculate the fault current and provide user indication in form of Trip command or/and in the form status message on the communication configured, here in this paper the communication protocol used is IEC61850 GOOSE.

\subsection{IED configuration manager.}

This is generally a PC based application provided by an IED vendor, with the IED which is capable of following:

$$
\begin{array}{ll}
\text { - } & \text { Configure device settings } \\
\text { - } & \text { Create and edit logic mapping. } \\
\text { - Configure device IEC61850 parameters, } \\
\text { interface/time zone } \\
\text { - Create user-definable language files }
\end{array}
$$

\subsection{IEC61850 Configurator}

This software may be a part of the above described IED configuration manager application or different $\mathrm{PC}$ application depending upon the vendor of the IED, which is capable of generating SCD, CID, ICD files for the IED which allows the user to configure the IEC61850 related parameters.

This application can:

specify the network structure and the properties of the individual device and subnets,

- Interconnect data objects of individual users and group such interconnections to form applications.

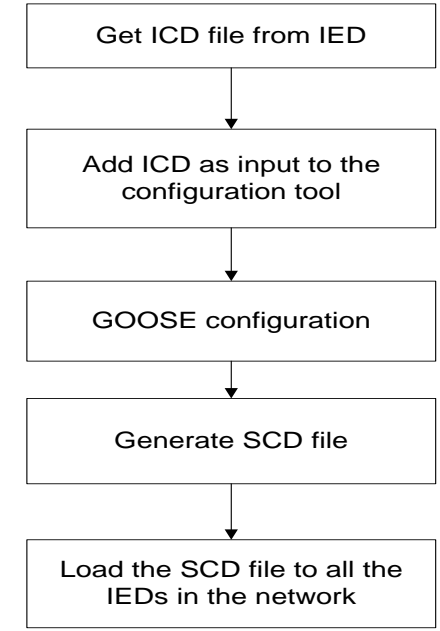

Figure 6: General flow of IEC 61850 configuration software.

\subsection{Omicron}

Omicron is a test tool capable of providing many test sets required for testing of protection devices. State sequencer module and the GOOSE module of the Omicron will for testing. The voltage to the input is provided by the State sequencer as well as the timing will be measured by the same module, the Omicron's GOOSE module is used subscribe the GOOSE message and map the status of virtual input to its BI status [5].

\section{GOOSE CONFIGURATION}

To test the GOOSE communication between the 2 IEDs under test one of the IED is configured as a publisher and the other as a subscriber. The publisher IED, which from now on will be called as IED1, is configured as the publisher with the status of one Trip element of the protection function (For 
simulating this, Binary input is used) in the GOOSE dataset. Whereas the subscribing IED i.e. IED2 is configured to

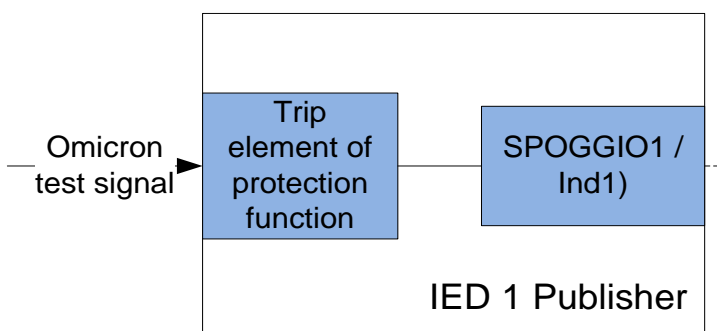

receive the GOOSE message and take necessary action as per the configuration.

Figure 7: GOOSE test setup

\subsection{Configuring IED 1 as GOOSE publisher.}

The IED's Trip element of protection function event can be mapped to publish GOOSE messages when energized with a test signal, so published as virtual signal. This signal is mapped into the GOOSE dataset using an IEC61850 configuration tool. The IED1 contains (among other logical devices) a logical device "CTRL", which serves as a container for all the control-related sub-functions.

For testing the protection function an event is simulated by Binary input of IED1, One of the logical nodes is "GGIO1" of the type GGIO (General purpose input output), which contains data information of Binary inputs. These data are mapped into the dataset of an IED which is sent out with the published GOOSE message. The GOOSE signal in the configuration tool is represented by the SPOGGIO logical node with indicate (Ind) as its data object., i.e. when there is a change in the voltage on the binary input of the IED1, the event is immediately sensed as protection event of the IED and GOOSE message is generated.

Table 1 shows GOOSE publisher configuration for IED 1. The data item Device_1/CTRL/SPOGGIO1/Ind1 visible under source in the "Signal Mapping" portion, is Binary input 1 , is part of the DataSet, which is published in the form of the GOOSE message by the IED.

Table 1: GOOSE publisher configuration

\begin{tabular}{|l|l|}
\hline \multicolumn{2}{|l|}{ Signal Mapping } \\
\hline Source & Description \\
\hline Device_1/CTRL/SPOGGIO1/Ind1 & $\begin{array}{l}\text { IED_1/Control/User SP } \\
\text { Outputs Block1/Single } \\
\text { Point Data 01 }\end{array}$ \\
\hline
\end{tabular}

\subsection{Configuring IED2 for GOOSE subscription}

The GOOSE message published by the IED1 is received by all the devices connected in the network. The received GOOSE message needs to be subscribed by the receiving IED. For subscription, the subscriber IED requires the control block reference, GOOSE ID, Application ID and broadcast MAC address of the publisher IED. In the IEC61850 configurator we only have to enter the subscribing signal in the destination device.

In the destination device, the signal Device_2/CTRL/SPIGGI01/SPCSO1 of IED 2 is selected for subscribing to the received GOOSE from IED 1 as shown in table 2 .

Table 2: GOOSE subscriber configuration

\begin{tabular}{|l|l|}
\hline \multicolumn{2}{|l|}{ Signal Mapping } \\
\hline Destination & Description \\
\hline & IED_2/Control/User \\
Device_2/CTRL/SPIGGI01/SPCS & SP Inputs \\
O1 & Block1/Single Point \\
& Data 01 \\
\hline
\end{tabular}

The GOOSE signal received by the subscribing IED is mapped to user output for processing. This user output can be a trip command or a binary output (BO) or even to an LED for visual indication.

Table 3: GOOSE configuration between IED1 and IED2

\begin{tabular}{|l|l|}
\hline \multicolumn{2}{|l|}{ Signal Mapping } \\
\hline Source (IED1) & Destination (IED2) \\
\hline $\begin{array}{l}\text { Device_1/CTRL/SPOGGIO } \\
\text { 1/Ind1 }\end{array}$ & $\begin{array}{l}\text { Device_2/CTRL/SPIGGI01/S } \\
\text { PCSO1 }\end{array}$ \\
\hline
\end{tabular}

The configurations mentioned in Table 3 are applied to the IEDs using the IEC61850 configurator utility.

Omicron test tool was used to energize the current input of the IED1 to demonstrate the IEC61850 GOOSE communication between the two IEDs. BI of IED1 is energized by providing a voltage above threshold limit from the Aux DC output of Omicron. The application of the DC voltage resembles event change in the IED1.

\subsection{Test for GOOSE timing}

The IEC61850 standard defines a timing constrain on the transfer of GOOSE message in a network, when a change on any member of the dataset is sensed. The transfer time definition as per the standard is as shown in the fig 8[7]. The transfer time is the sum of the processing time of the communication processor of publisher IED, the time taken for transmission and the processing time taken by the communication processor of the subscriber IED. 


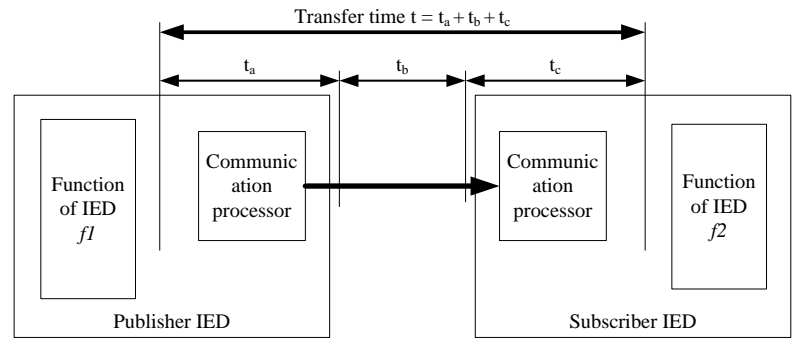

Figure 8: Definition of transfer time

\subsubsection{Round trip time (RTT)}

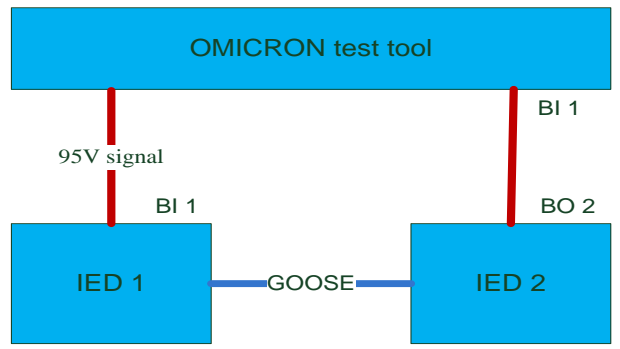

Figure 9: Block diagram for RTT

For the calculation of round trip time the setup is same as shown in figure 5 . The figure 9 gives block diagram of the round trip time test. The IED 1 is the publisher and IED 2 is the subscriber. The status of binary input 1 (BI 1) of IED1 is made a member of GOOSE dataset which is subscribed by the IED 2's signal SPCSO1. The SPCSO1 signal of IED2 is mapped to a user output for processing. In this test it is mapped to a BO 2 of IED2. This BO 2 is connected to the Omicron's BI 1 using copper cable.

To test the round trip time following test is conducted. A state sequencer tool of Omicron is used. This tool gives the time from the moment Omicron generates a voltage to the time an input is sensed on its BI. Three states are considered. State 1 is a delay of $1 \mathrm{sec}$, state 2 starts with application of the voltage to the BI 1 of IED 1 and concludes when an input is sensed on Omicrons BI 1 input. As soon as the state 2 concludes state 3 is started which is again a delay of $1 \mathrm{sec}$. The time difference between state 3 and 2 gives the round trip time.

\subsubsection{GOOSE publisher timing.}

The time taken to generate GOOSE message by the IED is calculated as follows: In this test procedure Omicron and IED are connected to an Ethernet switch. IED is the publisher and Omicron's GOOSE configuration module will subscribe to the GOOSE message from the IED. The status of binary input 1 (BI 1) of IED is made a member of GOOSE dataset. The SCD file generated by the IEC61850 configurator is loaded into the GOOSE configuration module of Omicron. This module allows user to map the member of the dataset to its binary inputs virtually. In this test the GOOSE dataset is mapped to BI 1 of Omicron.

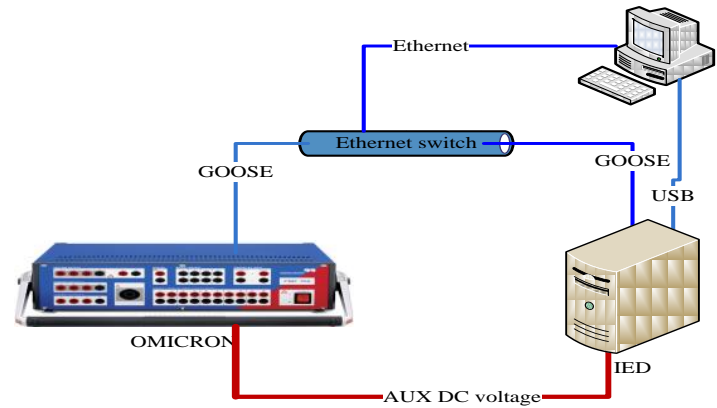

Figure 10: GOOSE publisher time test.

To test the time taken by the IED for generation of GOOSE and subscription by Omicron, state sequencer tool of Omicron is used. Same states are used as detailed in previous subsection.

\section{RESULTS \\ 6.1 GOOSE communication between 2 IEDs.}

The user output of the subscribing IED can be mapped to any of the peripheral via the user output. This can be mapped to a trip signal or any other protection related device. To visualize the effect of the GOOSE signal being subscribed in the laboratory environment the user output was mapped directly to an LED. The moment the current input of the publisher IED is energized, the LED on the subscriber IED is lit up indicating that the GOOSE communication between the IEDs is established. The contents of the GOOSE PDU, before and after the event has occurred, are shown in figure 11 and 12 respectively.

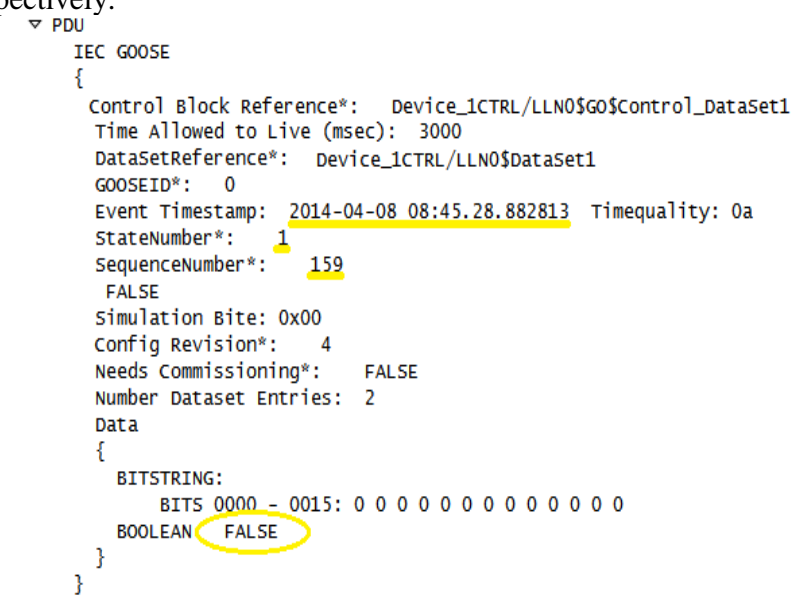

\section{Figure 11: GOOSE packet before event.}

In figure 11 the highlighted portions are timestamp, state number and sequence number of the GOOSE message before the event has occurred. The data in the GOOSE is also highlighted which is FALSE indicating that there is no surge of current sensed on the current input of the IED

After application of current from Omicron to the current input of the IED1 the change is detected and GOOSE message is published with the current status as shown in figure 11. The moment the applied current exceeds its threshold the data value turns to TRUE. As can be seen from the figure, the state number is increased and the sequence number is reset to 0 which is as per the standard description. 


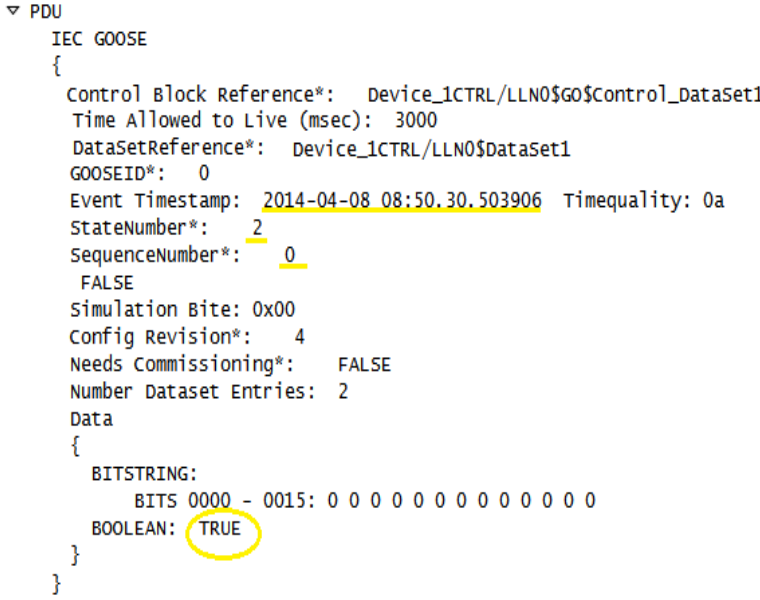

Figure 12: GOOSE packet after event.

\subsection{Round trip time}

Using the state sequencer a DC voltage above threshold is applied to the BI 1 of the IED1 which initiates a state 2 in the state sequencer. This causes an event and IED 1 generates GOOSE message. This message is subscribed by IED 2's binary output 2 (BO 2). As a result an input is sensed on the BI 1 of Omicron test tool. The moment the input on Omicron's BI 1 is sensed state 2 concludes and state 3 is started. The difference between state 3 and 2 is measured as $18.50 \mathrm{~ms}$, shown in figure 13. This end-to-end timing is inclusive of processing time of individual IEDs and Omicron such as input de-bounce, relay operation time, BI sensing time, etc.,

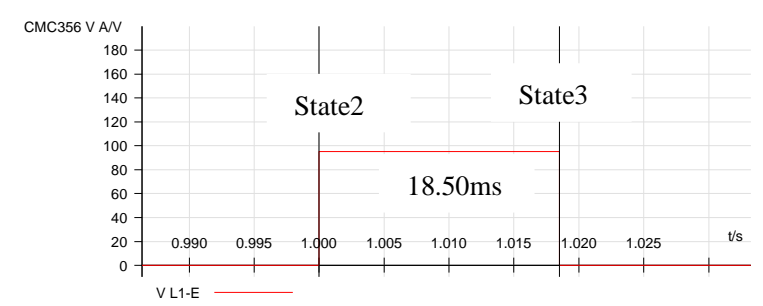

Figure 13: Round trip time graph.

\subsection{GOOSE publishing time}

Using the state sequencer a DC voltage above threshold is applied to the BI 1 of the IED which initiates a state 2 in the state sequencer. This causes an event and a GOOSE message is generated which is sensed by Omicron's GOOSE module. This GOOSE signal is mapped to Omicron's BI virtually. State 3 is started when input on Omicron's BI is sensed. The difference between state 3 and 2 gives the publishing time. The figure 14 shows the time graph of GOOSE message having only 1 data object in the dataset.

The time taken to publish GOOSE message having only 1 data object is $6.20 \mathrm{~ms}$. This end-to-end timing is inclusive of processing time of IEDs and Omicron such as inputs debounce, relay operation time, BI sensing time, etc.

Table 4: Timing analysis.

\begin{tabular}{|r|c|c|}
\hline $\begin{array}{l}\text { Sr. } \\
\text { No }\end{array}$ & $\begin{array}{l}\text { No. of data Objects in GOOSE } \\
\text { dataset }\end{array}$ & Time (ms) \\
\hline 1 & 1 & 6.20 \\
\hline 2 & 10 & 7.20 \\
\hline 3 & 20 & 8.80 \\
\hline
\end{tabular}

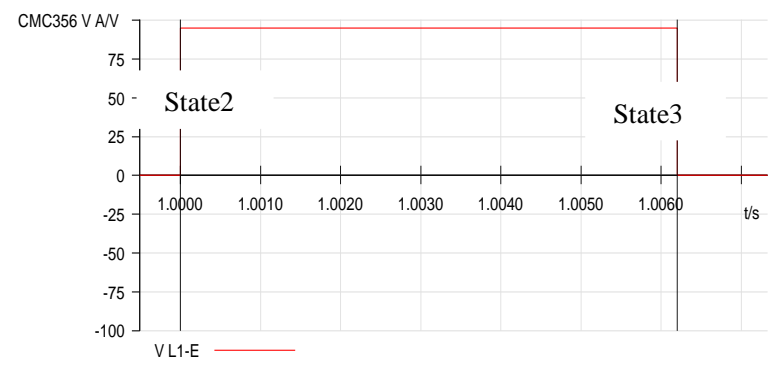

Figure 14: Timing graph of GOOSE message with 1 data object in dataset.

A number of tests were conducted by changing the number of data objects in a dataset. Three recordings are shown in table 4. It can be concluded from the table that, the number of data object in a dataset can affect the time needed for GOOSE publishing. The number of data objects in a dataset can be configured by user based on application priority.

\section{CONCLUSION}

In this paper, IEC61850 communication of a protective IED was tested successfully. Other functions of the IEDs can also be tested in similar fashion using similar setup. The IEC61850 GOOSE communication between 2 devices was successfully established and both the devices were able to publish and subscribe to the GOOSE messages. The timing measured in the tests mentioned above is not for functional verification of the IED. The successful integration of IEC61850 into the IED makes the IED convenient for implementing protection schema in the Substation Automation System. The GOOSE protocol uses standard Ethernet for high speed communication between IED to IED which can be easily realized in actual substation. VLAN tagging and other priority based network schema can be used to meet the timing requirement as defined by standard. The actual testing should be done according to the testing strategy detailed in the standard which is beyond the scope of this paper. The test procedure enlisted in the paper is to benefit the user and provide an insight on how basic testing of IED is carried out in the laboratory environment for understanding the GOOSE protocol.

\section{REFERENCES}

[1] R. E. Mackiewicz, Member, IEEE “Overview of IEC 61850 and Benefits “.

[2] M., Ikbal Ali, Sr. Assistant Professor, Senior Member, IEEE, "High-speed Peer-to-peer Communication based Protection Scheme Implementation and Testing in Laboratory", International Journal of Computer Applications (0975 - 8887) Volume 38-No.4, January 2012.

[3] http://blog.iec61850.com/

[4] C. Kriger, S. Behardien, J. Retonda-Modiya "A Detailed Analysis of the GOOSE Message Structure in an IEC 61850 Standard-Based Substation Automation System" INT J COMPUT COMMUN, ISSN 1841-9836 8(5):708721, October, 2013.

[5] Reference Manual, "CMC356 Hardware", OMICRON Electronics.

[6] IEC 61850-7 part series of the standard.

[7] H. Ito and K. Ohashi, Toshiba Corporation. "High Performance IEC 61850 GOOSE and Protection Relay Testing”, Japan. PacWorld Winter 2008. 